\title{
Les techniques, entre tradition et intention
}

\section{Georges Guille-Escuret}

\section{OpenEdition}

Journals

Édition électronique

URL : https://journals.openedition.org/tc/107

DOI : $10.4000 /$ tc. 107

ISSN : 1952-420X

\section{Éditeur}

Éditions de l'EHESS

\section{Édition imprimée}

Date de publication : 1 avril 2004

Pagination : 97-109

ISSN : 0248-6016

\section{Référence électronique}

Georges Guille-Escuret, «Les techniques, entre tradition et intention», Techniques \& Culture [En ligne], 42 | 2004, mis en ligne le 06 novembre 2007, consulté le 29 septembre 2022. URL : http:// journals.openedition.org/tc/107 ; DOI : https://doi.org/10.4000/tc.107

Ce document a été généré automatiquement le 29 septembre 2022.

Tous droits réservés 


\title{
Les techniques, entre tradition et intention
}

\author{
Georges Guille-Escuret
}

1 Sobre et apparemment limpide, la définition des techniques donnée par Marcel Mauss dans son Manuel d'ethnographie constitue la référence de base pour la technologie culturelle en France : elles seraient des « actes traditionnels groupés en vue d'un effet mécanique, physique ou chimique, actes connus comme tels" (Mauss 1967: 29). L'avantage principal de la proposition est de ne pas inféoder l'existence d'une technicité à la présence de l'outil, ce qui aurait pour conséquence d'exclure des relations directes entre l'homme et une matière «travaillée » (cueillette à mains nues, nage, etc.). Toutefois, le socle n'est pas sans défaut et, à bien y regarder, cette formule pose une multitude de problèmes entremêlés que l'envol impressionnant des recherches consacrées à la cognition tend aujourd'hui à faire ressortir, ne serait-ce qu'en réactivant le réseau complet des contradictions séculaires entre les démarches biologique, psychologique et sociologique.

2 D'une part, comme nous allons le voir, la constatation du caractère traditionnel de l'acte ne va pas forcément de pair avec celle de son efficacité physique ni avec celle de sa nature intentionnelle. D'autre part, ce ne sont pas les mêmes disciplines qui sont habilitées à confirmer ou à infirmer l'existence d'une tradition, celle d'un effet mécanique et celle d'une connaissance, car la tradition relève en principe d'une compétence en sciences sociales, tandis que la productivité technique renvoie pour sa part à un savoir dérivé de l'une ou l'autre des sciences de la nature. Quant au domaine de la connaissance -c'est-à-dire en l'occurrence de la correcte représentation d'une relation de cause à effet inhérente à la «technique » envisagée-, nul n'ignore que la psychologie, la philosophie et les «méta-sciences» en font une zone d'intense brouillard épistémologique bien avant que l'entrée en lice des questions sociologiques et historiques ne viennent compliquer encore les repérages.

Les enjeux d'une définition des techniques

3 Mais, après tout, cette imprécision est-elle vraiment regrettable ? Est-elle seulement embarrassante? Les définitions parfaites ne balisent en effet que des terrains archi- 
connus et l'on peut admettre que le discernement d'un aussi vaste champ de recherche que celui de la technologie supporte un certain flou, voire une hétérogénéité certaine, dès lors que s'affirme en même temps la résolution de cerner ainsi une série d'énigmes pour mieux les répertorier ensuite. La complaisance envers l'approximation sera alors justifiée par l'espoir de voir l'ensemble des analyses stimulé par la diversité des approches visant des phénomènes sinon identiques, du moins assez voisins pour s'éclairer mutuellement. Derechef, l'évocation des sciences de la « cognition » permet d'illustrer actuellement cette stratégie avec éclat.

Encore faut-il alors que cette disparité interne des aspirations n'ait pas pour premier résultat d'entretenir les confusions dans un cadre plus large, notamment en favorisant par ce biais le maintien ou la domination de tendances théoriques incapables de soutenir un débat sur la pertinence de leurs convictions initiales.

Or, l'identité de la technique n'est pas censée se restreindre à celle d'une élaboration intellectuelle progressive: elle ne se situe pas sur le même plan qu'un concept théorique destiné à rejoindre une réalité complexe, ou qu'un modèle perfectible. Le terme a pour fonction première de saisir un ensemble de phénomènes bruts qui apparaissent comme une des sources pures de l'information scientifique en anthropologie : rappelons qu'avec le fait biologique et le fait linguistique, et au même niveau d'importance qu'eux, le fait technique s'impose comme une des trois portes s'ouvrant directement sur la société (Guille-Escuret 1994). Ce sont aussi les trois accès privilégiés depuis plus d'un siècle pour fonder un évolutionnisme du social, comme si la ligne directrice du " progrès » culturel devait s'attacher à l'un d'eux comme à une sorte de tuteur. En contraste, un procès économique, une croyance religieuse, un agencement politique incorporent pour leur part une combinaison variable de déterminants vitaux, de gestes et de paroles: ce sont toujours des constructions composites, autant pour l'individu qui les met en pratique que pour celui qui les regarde à distance (sans négliger, bien entendu, le fait que l'un et l'autre les assemblent rarement de la même façon).

6 À cet égard, un regard d'ensemble sur l'histoire des grands débats anthropologiques amène vite deux constats.

7 D'abord, l'entrée technique est régulièrement négligée par rapport à ses homologues biologique et linguistique : à quelques exceptions près -on songe inévitablement au double sillage laissé par André Leroi-Gourhan et André-Georges Haudricourt-, la langue continue à être généralement présentée comme l'unique opposition concrète qui se soit dressée au cours de l'hominisation devant la dictature naturelle des causalités physiologiques ou génétiques.

8 Ensuite, la distinction des phénomènes élémentaires du biologique et du linguistique a été beaucoup plus affinée, épurée et contrôlée que celle du fait technique. Il en découle que, sur le plan théorique, la technologie est artificiellement maintenue dans une situation de perpétuelle attente, sa position méthodologique étant condamnée à se décanter à la suite des succès obtenus à partir des deux autres voies. Attente quasi désespérée, puisque ce sont des intuitions théoriques rivales qui s'attachent à celles-ci.

9 Autrement dit, bien qu'aucun type d'interprétation n'ose dénier explicitement aux techniques une puissance primordiale dans la genèse du social et de la condition humaine, elles passent presque systématiquement au second plan des tentatives de synthèse, devenant une sorte de réservoir informe et secret des questions cruciales que l'affrontement obsédant du biologisme et du « sémiologisme » (par là, on entend les 
explications axées sur l'efficacité propre des symboles) laisse en suspens. Avec la complicité inconsciente de ceux qui l'animent, l'antagonisme idéologique évacue ainsi «par réflexe » tout ce qui menace d'écorner son image d'universalité et d'omnipotence.

En ce sens, le brouillard qui plane sur la consistance du phénomène technique n'émane nullement d'une imprécision conjoncturelle, plus ou moins innocente. Il s'agit d'un véritable obstacle épistémologique, dans l'acception de Gaston Bachelard (1975), avec la résistance inconsciemment organisée qu'elle suppose de la part de tournures mentales socialement établies. Pour déceler les ressorts qui déclenchent cette force d'inertie, la définition de Mauss est alors un point de départ prometteur, précisément parce qu'elle est admise par les spécialistes de la technologie culturelle en dépit de sa flagrante faiblesse méthodologique.

«Techniques » troubles ou incomplètes : exemples

Afin de défricher la difficulté aussi largement que possible, le mieux est sans doute d'évoquer plusieurs situations où une "technique " déclarée échappe à cette délimitation classique. Ces situations sont très éloignées les unes des autres: cela réduira le risque de se concentrer prématurément sur un aspect trop étriqué d'une difficulté dont nous ignorons a priori l'étendue, même s'il est prévisible qu'elle tournera autour de la question d'une disparité contradictoire des «savoirs » associés à une même activité technique.

Les techniques des chimpanzés

De la célèbre pêche aux termites jusqu'au cassage des noix à l'aide d'une pierre, en passant par diverses observations moins spectaculaires, la primatologie de terrain a accumulé au cours des quatre dernières décennies une information considérable sur ce qu'il faut bien appeler les techniques des chimpanzés. Il est désormais établi que, d'une part, celles-ci sont marquées d'une population à l'autre par des variations régionales qu'aucune détermination strictement biologique ne semble susceptible d'expliquer et que, d'autre part, certaines d'entre elles se sont perpétuées à travers un grand nombre de générations (au-delà du siècle, parfois).

Il y a donc "culture" dans l'acception ethnologique du terme et non plus par l'entremise d'une reconstruction tendancieuse du concept par les zoologistes (Boesch et Boesch-Acherman 1991; McGrew 1992). Certains vont plus loin, parlant déjà de "cultures » au pluriel (Wrangham et al. 1994). On doit en outre, au moins à titre d'hypothèse, entériner l'existence d'une «tradition » embryonnaire (Joulian 1995; voir aussi le point de vue différent d'Abeg \& Thierry 1998).

Néanmoins, en l'absence d'un langage qui soit en mesure de le certifier, rien ne permet d'affirmer que l'efficacité de ces pratiques est « connue comme telle». D'autant que le principe de l'éthologie contemporaine, depuis sa fondation par Konrad Lorenz et Nikolaas Tinbergen, est de s'interdire toute spéculation sur la conscience de l'animal dans l'analyse de son comportement.

Les chimpanzés semblent apprendre exclusivement par imitation (ce qui n'exclut pas totalement des moments d'enseignement). Dès lors, la nature de la connaissance impliquée est un objet de recherche primordial que la définition de Mauss laisse malheureusement en amont. Contrairement à ce qu'un anthropologue pourrait alors imaginer a priori, la question ne se limite pas à une vision purement psychologique de la cognition : la formation de la société humaine est étroitement concernée et il suffit pour s'en convaincre de transposer le problème sur la stupéfiante lenteur de l'évolution de l'industrie lithique au Paléolithique inférieur et moyen. Est-elle concevable 
autrement que comme l'expression d'une tradition qui se perpétuerait hors du langage, grâce au support unique de l'imitation? De ce point de vue, paradoxalement, bien que résolue à immobiliser les comportements sous l'autorité des mots, la tradition orale apparaîtrait en accélérateur formidable du changement (Guille-Escuret s/presse).

Au sein d'une société humaine contemporaine, rien n'empêche le linguiste de saisir les mots en laissant de côté les pratiques. Par contre, le technologiste, lui, a infiniment plus de mal à appréhender les productions sans se référer aux discours qui les imprègnent ou les entourent. Cette dissymétrie ne contredit pas l'assertion selon laquelle la dimension technique correspond à une "matière première » de la réalité sociale : elle réclame seulement une attention méthodologique particulière et une distinction entre le geste culturellement commenté et le geste qui se précise hors de la parole. Surtout si la cognition se trouve à l'horizon de la recherche entreprise.

Désherbage et labour : une technique hybride des viticulteurs languedociens

La deuxième illustration montrera que la confrontation de la tradition et de la connaissance ne laisse pas intact le troisième paramètre invoqué par Mauss : l'efficacité matérielle. Dans les années 1970, le désherbant a pénétré dans quelques vignes languedociennes dans le but déclaré de se substituer aux labours.

18 Les premières expériences ont eu lieu sur des parcelles de terrain plat (où les tracteurs évoluaient facilement), sous contrôle d'un savoir agronomique institué. Puis, au fur et à mesure que les viticulteurs adoptaient la nouvelle technique, le désherbant s'est rendu de préférence sur les terrains où sa concurrence avec les usages traditionnels pouvait se révéler la plus utile : les coteaux escarpés, interdits au tracteur et déjà réservés jadis à la mule et à la houe parce que le cheval n'y "tenait " pas. Le désherbant s'y est substitué non pas à la charrue mais à l'araire ou au bigos (houe à deux dents), et il est devenu un moyen providentiel de maintenir en culture des vignes au rendement certes très faible mais donnant un vin fortement chargé en alcool.

19 Enfin, dans une troisième étape, l'usage du désherbant s'est étendu au terroir labouré et une stratégie agricole imprévue s'est rapidement répandue : au lieu de «croiser » les labours comme autrefois (c'est-à-dire de travailler successivement le sol dans une direction puis en sens perpendiculaire), ou de se contenter de l'épandage du désherbant, beaucoup d'exploitants ont décidé de croiser le labour et l'épandage, celuici prenant la place d'un des labours et non du labour dans son ensemble. Non seulement cette étrange idée n'est pas venue des techniciens qui avaient encouragé l'innovation, mais la valeur agronomique de la combinaison était à leurs yeux pour le moins douteuse.

20 Il est apparu que, par-delà les motifs exprimés (« la terre a quand même besoin de respirer ", etc.), l'hybridation des deux pratiques reposait avant tout sur la valeur sociale du labour dans la pensée des viticulteurs : renoncer totalement à ce procès de travail éminent -l'élaboration du vin étant renvoyée à la compétence ressentie comme externe de la coopérative - revenait à les priver d'une composante essentielle de leur auto-identification professionnelle (Guille-Escuret 1993).

21 La difficulté qui se lève devant la définition de Mauss est alors flagrante : si le labour et le désherbage sont assurément deux "techniques ", qu'en est-il de leur association quand se creuse un hiatus entre l'efficacité matérielle déclarée («connue comme telle ») et une efficacité culturelle à la fois plus complexe et mieux établie? Ne serait-il pas abusif, sur la base du constat de nécessité économique du désherbant, de renvoyer le labour hors du champ technologique vers un univers exclusivement symbolique? 
Sur cette voie, l'intervention polymorphe de la "tradition » ne serait-elle pas avilie et tronquée au détriment de l'analyse sociologique? Arrêtons momentanément ici ce questionnement que la situation suivante va encore élargir.

Le « poison » anti-vol dans les cultures de la forêt centrafricaine

22 Les horticulteurs de la forêt centrafricaine (les Ngbaka, les Ngando et leurs voisins) placent dans leurs jardins des "poisons » destinés à punir les visiteurs indélicats qui voudraient chaparder une partie de leur production: les Pygmées aka, pour commencer, ou des voisins peu scrupuleux. Le procédé se révèle assez dissuasif pour que certains Pygmées récemment convertis à une agriculture autonome regrettent de ne pas l'avoir à leur disposition (Guille-Escuret 1999). Et les villageois eux-mêmes sont persuadés de sa nocivité potentielle.

Pourtant, ces « poisons » cachés à travers la parcelle sont censés agir exclusivement sur les voleurs, sans qu'un contact physique soit nécessaire avec eux. Autrement dit, un observateur occidental tendra à ranger ces pratiques dans le registre de la sorcellerie, malgré le net démenti des horticulteurs. Mais, quand bien même des médecins et des agronomes analysant en profondeur lesdits « poisons » en arriveraient à la conclusion de leur totale inefficacité matérielle (au contraire de la puissance attestée d'autres produits enduisant, par exemple, les pointes de flèche), la technologie aurait-elle raison d'adresser le phénomène aux spécialistes de la classification et de se désintéresser de tels actes?

Répétons-le: l'effet de dissuasion provoqué par ce savoir contestable est très appréciable et l'efficacité de la croyance se matérialise indubitablement au bout du compte par une réduction sensible des vols. En conséquence, la description du système technique agricole des Ngbaka qui prendrait le parti de marginaliser son action en l'excluant de l'efficacité physico-chimique avérée s'exposerait à des distorsions dangereuses.

En d'autres termes, quand Mauss décide que la causalité incorporée par la technique doit être " connue comme telle », il laisse dans l'ombre une ambiguïté imperceptible à son époque mais sur laquelle les décennies suivantes ont fréquemment bronché et dont le relativisme a fait ses choux gras: le savoir du technologiste contre le savoir du technicien, avec la peine d'extraire cette confrontation d'un rapport d'autorité interculturel, c'est-à-dire très naïvement politique.

Méditation et invention

26 D'un point de vue plus général, il serait dommage de ne pas signaler brièvement deux types de cas qui déstabilisent la formule de Mauss par d'autres biais. Rappelons ainsi que le professeur commence son chapitre par des «techniques du corps» telles que la nage. Comment traiterons-nous donc une discipline spirituelle comme le yoga? Elle est traditionnelle et une partie de son efficacité matérielle est reconnue par tous, y compris par la médecine. Une partie seulement, car le physiologiste et l'adepte divergeront sur bien des assertions touchant à la frontière et aux interactions entre le matériel et le spirituel. Cet exemple a l'avantage de montrer que le technologiste n'est pas toujours en position de discriminer le savoir faux du savoir vrai chez ceux qu'il étudie.

Enfin, si l'on suit à la lettre la définition de Mauss, on sera contraint de décréter que l'expression « invention technique » est paradoxale, puisque le résultat d'une invention implique une rupture avec la tradition. Le progrès, l'innovation, l'emprunt et le transfert ne relèveraient donc pas d'une réalité technique proprement dite et ces 
changements appartiendraient à la sphère plus large du social. La proposition, même si elle conduit inexorablement la technologie vers un statut sociologique subalterne, n'est pas vraiment absurde: il resterait toutefois à en tirer toutes les conséquences méthodologiques et, pour commencer, à spécifier quel domaine prendra en charge l'examen scientifique des inventions qui, à terme, seront absorbées dans l'univers technique. Soit dit sans vouloir entamer un procès d'intention en bonne et due forme, une discussion qui, à ce sujet, se contenterait de mettre en présence le postmodernisme et le cognitivisme aurait à tout le moins quelque chose de suspect.

Les niveaux de la réalité technique

Recensons maintenant les contradictions en germe dans la définition de Mauss.

- La tradition ne s'accorde pas systématiquement avec une lucidité cognitive sur la technique.

- L'efficacité matérielle de la technique n'est pas forcément connue comme telle par les producteurs.

- Il peut y avoir une divergence profonde entre l'interprétation de l'efficacité matérielle reconnue par le producteur et celle que déduit le technologiste.

- L'efficacité physico-chimique de la technique peut être indirecte, l'intermédiaire étant une efficacité sociale garantie par la tradition.

Une première conclusion s'impose aussitôt : il n'y a pas trois paramètres (efficacité, tradition, connaissance) mais au moins quatre, car la connaissance est toujours entérinée à partir de deux points de vue incommensurables, celui du technicien et celui de l'enquêteur (sans négliger que dans le cas d'une société sans parole, simienne ou préhistorique, l'un des deux n'est pas directement accessible). Dans ces conditions, les techniques qui s'accommodent de la définition de Mauss sont celles qui ne sont pas saisies dans une perspective diachronique et dont l'efficacité est perçue pareillement par le producteur et par l'observateur. Elles existent, assurément, et forment même un corpus considérable dont la lacune est cependant évidente: une évacuation quasi automatique des risques de malentendus entre la technologie « pure » et la sociologie, moyennant l'exclusion des problèmes névralgiques.

C'est pourquoi nous avons dit «au moins » quatre paramètres: ce serait en effet une erreur que d'utiliser sans précaution l'image « du » point de vue de l'observateur, tant il est vrai que cette représentation masque une disparité essentielle des démarches derrière une appartenance commune à une culture proclamant son rationalisme. Nul n'ignore que la technologie culturelle s'oppose souvent aux jugements péremptoires d'une technologie issue d'une spécialité scientifique: un ethnographe ne voit pas les choses de la même façon qu'un médecin "normal » ou qu'un agronome "classique », pour saisir les exemples habituels.

Il s'ensuit que la technologie dite culturelle est régulièrement soumise à une tension désastreuse : ou bien soutenir la pertinence du savoir indigène contre une science étriquée, ou bien étudier la connaissance exotique en campant sur les certitudes pointues de la biologie, de la physique, etc. Dans le premier cas, elle renoncera à une compétence particulière devant l'efficacité technique et, dans le second, elle rétrécira jusqu'à l'absurde la délimitation d'une « efficacité sociale » qui imprègne et entoure le geste proprement dit. Piégée dans la vieille alternative du relativisme face au scientisme, elle avilira alors le savoir culturel en campant sur un savoir objectif lointain, ou bien elle contestera celui-ci en se faisant le défenseur forcément maladroit d'une connaissance exotique. Dilemme scabreux qui tend à invalider l'éventualité d'une problématique propre à la technologie culturelle. 
La technique, déductible du produit

recourt à une perspective historique qui lui permet de repérer les lacunes persistantes dans l'histoire de débats, ou d'indiquer des incohérences croissantes. Mais on a sans doute sous-estimé les enseignements potentiels d'une démarche complémentaire qui consisterait à comparer systématiquement -c'est-à-dire notamment à différentes échelles- le traitement de problèmes contigus ou de difficultés logiquement homologues par plusieurs domaines, thématiquement voisins ou non. Il arrive certes à l'épistémologie d'esquisser des confrontations interdisciplinaires, mais le plus souvent en considérant des ensembles si vastes et des difficultés si générales que les conclusions rejoignent plus aisément le terrain philosophique que la pratique scientifique. fait appel en ancrant la réflexion sur le constat des trois portes d'entrée dont dispose l'anthropologie. Le résultat ainsi obtenu sur la définition que Mauss donne des techniques -largement approuvée par les spécialistes, répétons-le- m'amène à conclure que cette formulation entérine un amalgame entre l'élément initial (disons du côté du geste) et la cohésion finale (comme le système technique complet), amalgame que d'autres disciplines ont pour leur compte davantage veillé à éviter, voire à empêcher.

La tâche d'une méthodologie constructive considérant les recherches se réclamant de la technologie serait donc de dissuader les argumentations de s'appuyer sur l'image subconsciente et fallacieuse d'une continuité phénoménologique entre différents degrés d'organisation du réel à observer, ne serait-ce que pour mettre fin aux concurrences absurdes qui s'ensuivent entre les approches disciplinaires dominant chacun de ces niveaux. En outre, sauf démonstration de l'absence objective de relations entre ces divers axes d'intérêt, cette finalité devra autant que possible être atteinte sans adopter la solution de facilité d'un éclatement de la technologie en petits secteurs étanches (qui ne se contrediraient plus parce qu'ils ne se parleraient plus).

En l'occurrence, contrairement à ce que l'on pourrait imaginer, cette ambition n'est pas inaccessible. Le défaut de la définition de Mauss, en effet, n'est pas d'impliquer des points de vue disciplinaires divergents mais bien de les juxtaposer sans leur offrir un support commun avec lequel ils pourraient se discerner mutuellement et spécifier leurs propres limites. En partie parce que ce grand ethnologue avait probablement pour préoccupation majeure de ne pas inclure une référence dangereuse à l'outil, ce qui d'ailleurs a assuré le succès de sa proposition aux yeux des sciences sociales. En partie, mais pas seulement. Si l'outil est effectivement impropre à fonder une reconnaissance synthétique des techniques, cela ne signifie pas qu'un autre concept ne serait pas en mesure de le faire.

Ce concept existe. Il est même évident et le professeur du Collège de France ne l'a sûrement pas manqué par accident. D'emblée, sa réflexion a été barrée par une aversion théorique qu'il mentionne ingénument quelques paragraphes après sa définition :

«L'erreur de Karl Marx est d'avoir cru que l'économie conditionnait la technique alors que c'est l'inverse " (Mauss $1967:$ 30). Socialiste de la première heure mais non marxiste (surtout dans les années trente où il donne les cours qui fourniront la substance du Manuel d'ethnographie), le neveu de Durkheim semble donc avoir conçu sa représentation des techniques en opposition à une image passablement déformée de 
l'économie: accepter de choisir entre une détermination de l'économie par la technologie et une inférence inverse revient à accréditer une aberration très éloignée de la pensée incriminée et la phrase touche moins l'économie au sens conçu par Marx lui-même que l'économisme prétendu marxiste aux yeux des adversaires du marxisme. Passons sur le mystère vertigineux de cette alternative grossièrement isolée et retenons seulement pour l'instant que Mauss en admet la pertinence : nous pourrons ainsi nous concentrer sur ce dont la technologie s'est vue soudain privée. Nul n'ignore en effet que Marx rend compte de l'économie autour du concept majeur de "production" (l'humanité, explique-t-il avec Engels dans une page fameuse de L'idéologie allemande, se distingue de l'animalité à partir du moment où elle produit ses moyens d'existence). Or, cette notion-là intéresse essentiellement les deux champs de recherche arbitrairement placés ici en situation de concurrence : il y a des techniques sans outil, d'accord, mais elles sont en revanche inconcevables sans produit.

Les techniques sont des actes groupés en vue d'un produit. Pas plus. L'efficacité matérielle, la dimension traditionnelle et le caractère intentionnel y sont des variables : elles entrent ou non dans sa composition et, quand elles le font, c'est selon une multitude de modalités qu'il importe de décrire convenablement. La noix cassée par le chimpanzé est un produit. L'absence de voleurs sur une parcelle ngbaka "protégée » en est un autre, d'une nature différente. La compatibilité revendiquée du désherbage et du labour sur une vigne équivaut à l'addition de deux produits répondant à des besoins sans commune mesure.

À ce point, l'imbroglio historique se détache enfin de la difficulté méthodologique sousjacente qu'il a imprégnée, voilée et finalement entretenue. Mauss n'a pas tort de considérer que la technique conditionne l'économie, si et seulement si on entend par là qu'une «matière première » conditionne la construction qu'elle contribue à édifier. L'ensemble est conditionné par ce qu'il incorpore totalement ou partiellement. Néanmoins, dans cette perspective, l'économie conditionne aussi à long terme et par des voies particulières ce qu'elle inscrit dans sa formation. Ce parcours à double sens est justement celui qu'emprunte Marx, lequel s'intéresse plus que tout autre théoricien aux relations qui unissent l'efficacité technique et l'efficacité sociale dans un procès de travail (Balibar 1980). En rejetant donc un économisme mal identifié, Mauss -qui, à l'instar de son oncle, n'a jamais cessé d'être irrésistiblement attiré par l'horizon d'une psychologie sociale en laquelle le structuralisme s'est ensuite reconnu sans réticences'est donc détourné du couple de concepts destinés à servir une sociologie complète : production et produit.

Que reste-t-il ensuite de la difficulté méthodologique? En dépit des apparences, elle n'est pas intacte. Envisageons la plus prévisible des objections : notre argumentation a seulement déplacé le problème, tous les malentendus demeurant menaçants, au sein de la définition du produit, entre les problématiques privilégiant respectivement l'effet matériel, la tradition sociale et la cognition. Bien qu'elle ne soit pas fausse, cette observation devra être nuancée. D'abord, le rôle bien compris de la méthodologie n'est évidemment pas de résoudre des problèmes scientifiques, sauf à confondre le traitement des informations et leur recueil : son ambition se borne à délimiter les discussions de manière à leur permettre d'avancer et d'aboutir. Elle a à traquer, parmi les incohérences de l'expression scientifique, celles qui risquent de ruiner à l'avance les débats. 
41 La confrontation de l'efficacité matérielle, de la tradition et de la cognition ne sera pas inchangée quand elle se transportera dans le cadre du produit: il ne s'agit plus d'une liste de critères que la technique doit additionner pour être avérée en tant que telle. Il y aura technique quand une science, « naturelle » ou non, « humaine » ou non, « sociale » ou non, saura désigner un produit grâce à sa compétence propre. La magie que réfute l'ingénieur pour son compte, l'ethnologue est en droit de l'admettre sur son domaine. Partant, l'assemblage de techniques élémentaires en systèmes complexes n'exige plus comme préalable que les sociologues, les psychologues, les biologistes et les ingénieurs aient résolu leurs désaccords - gain très appréciable, on en conviendra- puisqu'aucun d'entre eux n'aura plus à défendre sa vision de la technique dans un jeu d'appropriation créant ex nihilo des concurrences insolubles vite récupérées par des antagonismes philosophiques séculaires. À l'exemple d'une linguistique dont le phonologiste ne prétend pas exclure le sémiologiste, pourquoi ne pas rêver à une technologie qui associerait sans les mélanger l'étude de plusieurs complexités complémentaires? Aurait-on tellement tort en s'inspirant conjointement de Marx, Leroi-Gourhan et Haudricourt (au moins) pour penser que le chemin qui conduit d'une percussion simple à une lourde machine est mieux suivi quand on n'oublie pas celui qui va du geste au discours, celui qui va de l'expérience pratique à la connaissance formulée, celui qui va du rapport homme/matière au rapport homme/homme, celui qui va du produit simple à l'ensemble de produits et celui qui va du produit à l'organisation sociale? Sans oublier celui qui va de l'alternative technique à la contradiction culturelle. Faut-il chaque fois attendre de découvrir que le rite magique n'ayant ici ou là aucune efficacité matérielle sur le cycle annuel d'une culture garantissait en fait son insertion dans l'écosystème à l'échelle d'une génération humaine pour subodorer la complémentarité souhaitable de l'agronomie, de l'écologie et de l'ethnologie par-delà une définition rigide et préfabriquée de l'essence profonde des techniques?

Déclarer que la formule de Mauss a aujourd'hui l'inconvénient de perpétuer un réseau de compétitions irrationnelles en juxtaposant des approches dissemblables ne saurait passer pour une dévaluation de son apport historique: en science, ce qui a été un moteur est toujours destiné à devenir un frein. Malgré son austérité pointilliste et, avouons-le, peu enthousiasmante, l'exercice méthodologique ne serait pas une perte de temps s'il facilitait un tant soit peu le dépassement de clivages arrivés au stade de la stérilité en préparant des discussions dont on ne parviendrait plus à prédire l'issue. En technologie, chaque champ de recherche est en mesure de modéliser seul des systèmes homogènes qui comprennent des produits d'un même type. La coopération de plusieurs champs de recherche dans la conception de systèmes techniques hétérogènes où seraient repérées les interactions entre diverses formes de produits est la condition d'un fonctionnement scientifique régulier de la technologie culturelle, où les ethnologues sont les principaux demandeurs sans réclamer une quelconque préséance. 


\section{BIBLIOGRAPHIE}

Abbeg, Ch., \& Thierry, Bernard

1998. «L'origine des traditions chez les singes", pp. 137-150, in A. Ducros, J. Ducros \& F. Joulian

(eds), La culture est-elle naturelle ?. Paris : Errance.

Bachelard, Gaston

1975. La Formation de l'esprit scientifique. Paris : J. Vrin.

Boesch, Christhophe \& Hedwige Boesch-Acherman

1991. « Les chimpanzés et l'outil », La Recherche 233 : 724-731.

Guille-Escuret, Georges

1993. « The social weight of plowing in the vineyards of Les Corbières (Languedoc) », pp. 214-226, in P. Lemonnier (ed.), Technological Choices : Transformation in material cultures since the Neolithic.

London : Routledge.

1994. Le Décalage humain. Le fait social dans l'évolution. Paris : Kimé.

1999. « La révolution agricole des Pygmées aka », L’Homme 147 : 105-126.

2000. «L'évolution accélérée par la tradition?», Primatologie.

Joulian, Frédéric

1995. « Mise en évidence de différences traditionnelles dans le cassage des noix chez les chimpanzés (Pan troglodytes) de la Côte-d'Ivoire, implications paléoanthropologiques ", Journal des Africanistes 65 : 57-77.

Mauss, Marcel

1967. Manuel d'ethnographie. Paris : Payot (1ère édition 1947).

McGrew, W.C.

1992. Chimpanzee material culture. Implications for Human Evolution. Cambridge : Cambridge

University Press.

Wrangham, R. W., McGrew, W.C., De Waal, F.B.M. \& P.G. Heltne (eds)

1994. Chimpanzee Cultures. Cambridge (Mass.) : Harvard University Press.

\section{RÉSUMÉS}

Bien qu'unanimement admise par les chercheurs concernés, la définition des techniques donnée par Marcel Mauss recèle un défaut que les recherches contemporaines rendent de plus en plus apparent et qui, par conséquent, ne peut manquer de devenir embarrassant. Ses trois critères essentiels - tradition, efficacité, connaissance- ne sont pas toujours compatibles. Cette brève réflexion tente d'établir les raisons de cette difficulté et propose une définition qui se place en deçà du débat théorique qu'elle dissimule.

Techniques, between tradition and intention. Though Marcel Mauss's definition of techniques is unanimously accepted by researchers, contemporary studies clearly reveal a flow which becomes obviously embarrassing. The three criteria of tradition, efficiency and knowledge are not always compatible. This short article tries to establish the reasons of this problem, proposing a definition to go beyond the theoretical debate. 
Las técnicas, entre tradición e intención. Aunque los investigadores unánimos la aceptan, la definición de las técnicas por Marcel Mauss ocultaba un defecto que los estudios contemporáneos hacen cada vez más evidente y que forzozamente se esta volviendo entorpecedor. Sus tres criterios esenciales - tradición, eficacia, saber consciente- no son siempre compatibles. La reflexión del autor procura evidenciar las raíces de esta dificultad y propone una definición situada más acá de un debate disimulado.

INDEX

Mots-clés : efficacité, épistémologie, Mauss (M), techniques, tradition

\section{AUTEUR}

\section{GEORGES GUILLE-ESCURET}

UMR 5196, Techniques et culture, CNRS, 27 rue Paul Bert, 94204 Ivry-sur-Seine Cedex 